\title{
Sensorimotor Cortex
}

National Cancer Institute

\section{Source}

National Cancer Institute. Sensorimotor Cortex. NCI Thesaurus. Code C154777.

The region of the brain that consists of the precentral and postcentral gyri and is involved in somatosensory and motor functions. 